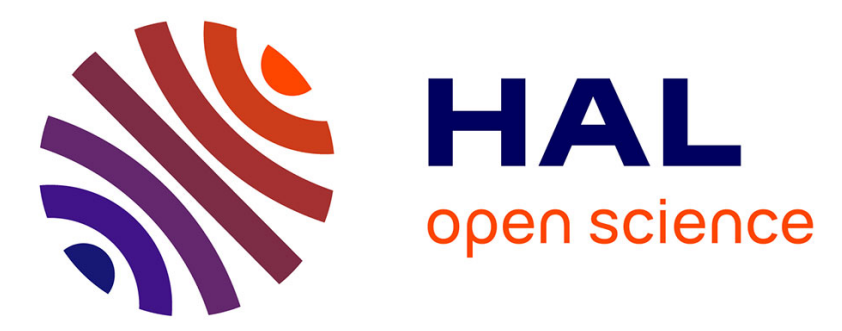

\title{
Molecular, clinical and neuropsychological study in 31 patients with Kabuki syndrome and KMT2D mutations
}

Natacha Lehman, Anne-Claire Mazery, Antoine Visier, Clarisse Baumann, Dominique Lachesnais, Yline Capri, Annick Toutain, Sylvie Odent, Myriam Mikaty, Cyril Goizet, et al.

\section{To cite this version:}

Natacha Lehman, Anne-Claire Mazery, Antoine Visier, Clarisse Baumann, Dominique Lachesnais, et al.. Molecular, clinical and neuropsychological study in 31 patients with Kabuki syndrome and KMT2D mutations. Clinical Genetics, 2017, 92 (3), pp.298-305. 10.1111/cge.13010 . hal-01560204

\section{HAL Id: hal-01560204 \\ https://u-bourgogne.hal.science/hal-01560204}

Submitted on 11 Jul 2017

HAL is a multi-disciplinary open access archive for the deposit and dissemination of scientific research documents, whether they are published or not. The documents may come from teaching and research institutions in France or abroad, or from public or private research centers.
L'archive ouverte pluridisciplinaire HAL, est destinée au dépôt et à la diffusion de documents scientifiques de niveau recherche, publiés ou non, émanant des établissements d'enseignement et de recherche français ou étrangers, des laboratoires publics ou privés. 
Molecular, clinical and neuropsychological study in 31 patients with Kabuki syndrome and $K M T 2 D$ mutations

Natacha Lehman 1, Anne Claire Mazery 2, Antoine Visier 3, Clarisse Baumann 4, Dominique Lachesnais 4, Yline Capri 4, Annick Toutain 5, Sylvie Odent 6, Myriam Mikaty 6, Cyril Goizet 7, Emmanuelle Taupiac7, Marie Line Jacquemont 8, Elodie Sanchez 1, Elise Schaefer 9, Vincent Gatinois 1, Laurence Faivre 10, Delphine Minot 10, Honorine Kayirangwa 2, Kim-Hanh Le Qang Sang 2, Nathalie Boddaert 11, Sophie Bayard 12, Didier Lacombe 7, Sébastien Moutton 7, Isabelle Touitou 1-13, Marlène Rio 2, Jeanne Amiel 2, Stanislas Lyonnet 2, Damien Sanlaville 14, Marie Christine Picot 3, David Geneviève 1

1 - Département de génétique médicale, maladies rares et médecine personnalisée, centre de référence anomalies du développement et syndromes malformatifs, Unité Inserm U1183, Hôpital Arnaud de Villeneuve, Université Montpellier, CHU Montpellier, France.

2 - Service de Génétique, Hôpital Necker-Enfants Malades, AP-HP et INSERM UMR 1163, Paris Descartes-Sorbonne Paris Cité University, Institut Imagine, Paris, France.

3 - Département de l’information médicale, CHRU Montpellier, France.

4 - Service de génétique médicale, Hôpital Robert Debré, Paris, France.

5 - Service de génétique, CHU Tours, France.

6 - Service de génétique clinique, Hôpital Sud CHU Rennes, Université de Rennes 1, CNRS UMR 6290 Rennes.

7 - Service de génétique médicale, CHU Bordeaux, INSERM U1211, Université de Bordeaux, France. 
8 - Unité de génétique médicale, CHU La Réunion, site GHSR, France

9 - Service de génétique médicale, Institut de Génétique Médicale d’Alsace, Hôpitaux

Universitaires de Strasbourg, Strasbourg, France.

10 - Centre de Génétique et Centre de Référence Anomalies du développement et Syndrome Malformatifs, CHU de Dijon et Université de Bourgogne, Dijon, France.

11 - Service de radiologie pédiatrique, Hôpital Necker Enfants Malades, France.

12- Laboratoire Epsylon, EA 4556, Université Paul Valéry Montpellier 3, Montpellier, France.

13 - Laboratoire de génétique des maladies rares et maladies auto inflammatoires, Montpellier, France.

14 - HCL, Service de génétique ; Centre de Recherche en Neurosciences de Lyon, Inserm U1028, UMR CNRS 5292, GENDEV Team, Université Claude Bernard Lyon 1, Lyon, France.

Corresponding author: Natacha Lehman

Département de génétique médicale, maladies rares et médecine personnalisée, centre de référence anomalies du développement et syndromes malformatifs, Unité Inserm U1183, Hôpital Arnaud de Villeneuve, Université Montpellier, CHU Montpellier, France.

Tel: +330617437801

Fax: +330467336052

Email: natacha.lehman@gmail.com

\section{CONFLICT OF INTEREST}

The authors declare no conflict of interest 


\section{ACKNOWLEDGEMENTS}

We thank the families and the French Kabuki association (http://www.syndromekabuki.fr/) for their participation in this study. Part of this work was funded by the French Ministry of Health (Programme Hospitalier de Recherche Clinique national AOM 07-090), Fondation Maladies Rares and the French Kabuki Association. 


\section{INTRODUCTION}

Kabuki syndrome (KS; OMIM 147920) is a rare genetic syndrome with an estimated incidence of $1 / 32000$ live births ${ }^{1}$. KS was first described in $1981^{2,3}$ and is characterized by the combination of five major clinical features, namely, visceral malformations, preand post-natal short stature, characteristic facial features, abnormal dermatoglyphic pattern including fetal finger pads and intellectual disability. Additional features observed are seizure, deafness, microcephaly or vision impairment. Mutations in two genes have been reported to be responsible for KS: lysine-specific methyltransferase 2D (KMT2D; OMIM 602113), the major gene involved in about $70 \%$ of patients ${ }^{4,5}$, and lysine-specific demethylase 6A (KDM6A; OMIM 159555), occurring in less than 5\% of patients ${ }^{6,7}$.

Since the first observation of KS, mild intellectual disability has been described in these patients, and large series emphasizing clinical features as well as molecular screening have been reported ${ }^{8,9,10}$. However, we have few data on the characteristics of cognitive functioning in KS. According to Niikawa et al. (1988), up to $90 \%$ of diagnosed patients have a Full Scale Intelligence Quotient (FSIQ) less than $80^{1}$. Oral motor, speech and language impairment has been investigated by Morgan et al. in a molecular heterogeneous cohort of $16 \mathrm{KS}$ patients ${ }^{11}$. Other studies focusing on FSIQ have shown that most patients feature weak visuospatial construction but relative strengths in verbal reasoning abilities ${ }^{12}$. However, these studies involved a limited group of participants and used different tests, therefore revealing only a small part of the puzzle of the cognitive impairment in KS patients. Clinical correlates of ID remain unknown.

No research has specifically studied the cognitive profile of a significant number of patients by using a standardized battery for evaluating FSIQ. Here we aimed to improve 
knowledge of the intellectual profile of patients with KS. We performed an intellectual assessment of $31 \mathrm{KS}$ patients with KMT2D mutation, examined a particular neuropsychological pattern and searched for genotype-phenotype correlations.

\section{MATERIAL and METHODS}

\section{Patient recruitment, clinical and molecular study}

Clinical data from 31 patients with KS and KMTD2 mutation were collected in the context of the French research program PHRC AOM-09-070 (ClinicalTrials.gov NCT01314534; Ethical approvals: Comité de protection des personnes île de France 2). For the present study, we extracted data regarding age, gender, presence of seizure, microcephaly (occipitofrontal circumference < -3 SD), congenital heart malformation, ophthalmological anomaly (ranging from abnormal eye refraction or strabismus to retinitis coloboma), deafness (sensorineural or conductive), and cleft palate as well as cognitive ability measures. Table 1 summarizes the main data obtained from the PHRC. Molecular screening of the KMT2D gene involved direct sequencing of exons and exon-intron junctions by using Sanger and next-generation sequencing methods. Molecular results from this cohort are published in Bögershausen et al. (2016) ${ }^{13}$. To observe a genotype-phenotype correlation, we classified putatively truncating mutations (i.e., premature stop codon, frameshift, and splice-site mutation) or non-truncating mutations (i.e., missense).

\section{Intellectual assessment}

The Wechsler Intelligence Scale for Children, Fourth Edition ${ }^{14}$ (WISC-IV) has been used to evaluate intellectual ability and cognitive processing. It contains 10 core tests 
and five supplemental subtests. The subtests are grouped as composite quotients into four indexes. One index evaluates the language: Verbal Comprehension Index (VCI). The Perceptive Reasoning Index (PRI) measures the ability to interpret and organize visual material. The Working Memory Index (WMI) measures immediate memory and the ability to concentrate. The Processing Speed Index (PSI) measures the ability to process visually perceived material quickly, with concentration, and eye-hand coordination $^{14}$. The combination of these indexes allows for calculating a Full Scale Intellectual Quotient (FSIQ) ${ }^{14}$.

For each child, we compared the indexes of the WISC-IV, namely, the VCI, PRI, WMI and PSI, two by two. To determine the significance of the difference between the indexes, we referred to the WISC-IV manual ${ }^{14}$. In this manual, the significance of the difference is defined by $\mathrm{p}<0.05$.

\section{Statistical analysis}

Baseline characteristics are described with frequency (percentage) for categorical variables and mean (SD) or median (interquartile range) for continuous variables after assessment for normal distribution by Shapiro-Wilk test. Quantitative variables with a Gaussian distribution were compared by Student $t$ test and Mann-Whitney test otherwise. For categorical variables, chi-square or Fisher exact test was used. $\mathrm{P}<0.05$ was considered statistically significant. Statistical analyses involved use of SAS v11 (SAS Inst., Cary, NC).

\section{RESULTS}

\section{Participants and features}


FSIQ was evaluated in 31 children with KS syndrome and mutation in KMT2D (16 boys; mean age 11.63; range 6 to 15 years and 15 girls; mean age 11,33 range 6 to 16 ). The mean FSIQ was 57.4 (range 40-103). The features considered to alter the FSIQ as well as the type and localization of mutations are in Table 1 (Fig. 1 represents a schematic location of mutations at the molecular level). WISC-IV results are in Table 2. Detailed data obtained from our serie are in Table S1.

\section{Association between FSIQ and clinical features (Table 3)}

Mean FSIQ was significantly lower with than without ophthalmologic anomalies. It did not differ by presence of cleft palate, microcephaly, heart malformation, hearing impairment, or epilepsy.

In addition, mean VCI and PRI was reduced with an ophthalmologic anomaly. Mean PSI was lower for male than female patients and mean PRI was higher for patients less than 10 years old than older patients.

\section{Genotype-phenotype correlation}

Mean FSIQ was lower with a putative truncating mutation than a mutation leading to a substitution ( $\mathrm{p}=0.05)$ (Table 3$)$ and VCI was lower with a truncating than substitution mutation ( $\mathrm{p}=0.049)$. Exon 48 was the most frequently mutated exon in KS patients from this series (table S1). Mean PRI was higher with the mutation localized in exon 48 versus other exons $(p=0.020)$. The frequency of ophthalmic abnormality did not differ between patients with than without a truncating mutation or with than without a mutation in the exon 48. In addition, there is no difference in IQ or indexes when 
mutations are non-truncating and are localized in exon 48, compared to non-truncating mutations located in other exons.

\section{Heterogeneous Intellectual Profile (HIP) in KS patients with KMT2D mutations}

We observed a discrepancy between the indexes of the WISC IV in our cohort. Scores were higher for VCI and WMI than PRI and PSI (Fig. 2).The most important difference was between WMI and PRI. The WMI was significantly higher than the PRI for $42 \%$ $(13 / 31)$ of children $(p<0.05)$. We propose that this particular neuropsychological phenomenon, WMI>PRI, be called HIP.

In addition, mean (SD) FSIQ was significantly higher for KS patients with than without HIP (67 [16] vs 51 [9], p<.001). We found no significant link between HIP and clinical features or types of mutation.

Mean VCI and WMI scores were significantly higher for HIP KS patients than KS patients with homogenous FSIQ scores (81 [17] vs 93 [12] and 64 [13] vs 61 [10], $\mathrm{p}<.05$ and $\mathrm{p}<.001$, respectively. Scores were higher for KS patients with HIP than other patients for the following subtests: similarities, digit span, vocabulary, letter number, comprehension, information, and arithmetic (Table 4).

\section{Association between subtest scores and clinical features (Table 4)}

Ophthalmologic anomaly was the only clinical feature associated with a difference in subtest scores. Indeed, scores for picture concept, comprehension, and picture completion tests were reduced with an ophthalmologic anomaly. 


\section{DISCUSSION}

KS is a rare genetic disease, and characteristics of its cognitive profile are still little known. A good knowledge of the cognitive profile may be helpful to develop tailored learning and is essential in the care of children with intellectual disability. In this study, we aimed to improve knowledge of the cognitive profile of children with KS, taking into account several features observed in KS (i.e., epilepsy, microcephaly, congenital heart malformation as well as visual or hearing impairment) that may affect IQ. We also investigated the type and localization of the mutation in the KMT2D gene to examine any genotype-phenotype correlation. Mean FSIQ and VCI scores were lower for patients with a truncating mutation than other types of mutations. In addition, mean FSIQ, VCI and PRI scores were lower with visual impairment than normal vision. We identified a discrepancy in indexes characterized by high WMI and VCI and low PRI and PSI. We emphasize the importance of early identification and intensive care of visual disorders in KS.

\section{IQ profile in KS patients}

In accordance with the literature, we found an important heterogeneity of global cognitive functioning in KS patients ${ }^{10}$, with intellectual ability ranging from normal to severe disability. However, most children showed moderately impaired IQ. Surprisingly, we observed a recurrent discordance between the index scores of the WISC-IV tests in our KS patients. Indeed, patients had strengths in VCI and WMI and weaknesses in PRI and PSI. We defined HIP based on a significantly better performance in the WMI than PRI. We chose these two indexes because their discrepancies were the greatest among all indexes. After evaluating the significance of the WMI-PRI difference, about $40 \%$ of our KS patients showed HIP.

Neither the features studied nor the type of mutations we evaluated seemed linked to HIP. However, FSIQ was higher for patients with than without HIP. A discrepancy in IQ indexes 
was shown for patients with William syndrome. Indeed, the authors highlighted a relative verbal strength in comparison to significant visuo-spatial weakness ${ }^{15}$. In our series, some of our patients may have had a too-low score for observing a difference between the indexes. Conversely, when results of the IQ test are too low, children graduate with a homogeneous score, which reflects a floor effect. This floor effect may hide the HIP profile in KS patients with severe intellectual disability.

Nevertheless, many children with KS have better abilities in working memory and vocabulary comprehension than in speed processing and perceptive reasoning. For these children, we suggest emphasizing learning at the auditivo verbal channel rather than the visual channel.

Scores were high on the similarities and digit span subtests for KS patients but were low on the block design and coding subtests. In addition, working memory seems to a strength of KS patients, which might be also an important point to promote during learning. Nevertheless, the heterogeneity in our sample highlights the importance of considering the cognitive profile of each child with KS.

\section{Features modifying IQ in KS patients}

We observed a link between demographic data (gender, and age) and some primary index scores of IQ. Indeed, the PSI was 10 points higher in girls than boys. The variability in IQ due to gender differences in genetic syndromes was suspected in children with 22q11.2 deletion. However, these data are still controversial ${ }^{16}$.

In addition, the PRI was lower for children older than 10 years than younger children. This result raises the question of whether a change in schooling might explain a decrease in PRI score after age 10. Indeed, in France, young children with KS share the same schooling as other children. Usually at age 6 to 8 years, they might be educated in a special school. 
Another hypothesis could be the acquisition of skills plateaus with age for KS patients, which therefore increases the gap compared to the reference population. The evolution of IQ in KS children could be studied in a longitudinal study to observe any IQ decline with increasing age and if this decline is linked to schooling.

Microcephaly, congenital heart malformation, and hearing impairment did not seem to affect IQ and the indexes for our cohort. In addition, mean FSIQ did not differ between KS patients with or without seizures, unlike the results from Tsai et al. in $2015^{17}$.

Among the clinical features we studied, only ophthalmologic anomaly seemed linked to altered IQ. All visual impairments, namely strabismus or refractive disorders (i.e., myopia and strabismus), have been corrected, except for retinal malformation (i.e., coloboma). In our population, IQ was significantly lower (10 points) for children with than without ophthalmologic anomalies.

Only the VCI and PRI seemed to be affected by visual impairment. Some tasks administered in the WISC-IV depend on the visual channel. Indeed, the use of the visual channel is significant in the PRI subtest but not the VCI subtest. When investigating the different subtests used to calculate the VCI and PRI, we found that patients with an ophthalmologic anomaly had low scores for picture concept and picture completion, tests known to use the visual channel. However, these patients also had low scores on the comprehension test, which does not require the visual channel. The presence of ophthalmologic anomalies might also affect intellectual functioning in KS patients with $K M T 2 D$ mutation, even in a field that does not seem directly linked to vision. Visual impairment in KS children is frequently undetected $^{18}$. We strongly recommend early screening and intensive care of ophthalmological anomalies in KS patients. Because only ophtalmological abnormalities seemed to modulate IQ, further research could explore the type of anomaly and whether treatment of the defect affects outcome. Our study has some limitations. The memory assessed in the WISC-IV study 
is only working memory. It would be useful to assess memory in depth with specific memory tests. It would be interesting to observe the evolution of the results of these children as they age in a longitudinal study with an in-depth evaluation of the education and medical interventions offered to them, especially for schoolage children.

\section{Genotype-phenotype correlation}

We found IQ 10 points lower for KS patients with than without truncating mutations, with VCI the most affected index. It has been previously observed that truncating mutations might be responsible for a more severe phenotype than non-truncating mutation. Indeed, a prediction of the effect of mutation on TSC1 and TSC2 proteins and intellectual ability was reported in tuberous sclerosis complex ${ }^{19}$. Truncating mutation in TSC1 leading to a nonsense-mediated decay was associated with more severe intellectual disability as compared with other mutations. It is tempting to speculate that absence or a short form of the protein encoded by $K M T 2 D$ leads to a more severe loss of function. Truncated $K M T 2 D$ protein would lead to major modifications of the methylation marks on targeted histones and therefore to more difficulties regarding intellectual functioning.

As well, the PRI was higher for patients with than without mutation in exon 48. Mutations in the exon 48 did not affect a specific domain of the protein (Figure 1). In addition, we found no link between ophthalmic anomalies and mutation in exon 48 or truncating mutation.

To conclude, our study contributed to improve our knowledge regarding the cognitive profile of children with KS. Approximately half of our KS patients showed a discrepancy in indexes we call HIP. HIP is characterized by strengths in WMI and weaknesses in PRI. The heterogeneity of the intellectual profiles highlights the necessity of neuropsychological assessment for each child. In addition, we highlighted a correlation between visual deficiency 
and reduced IQ. We emphasize the need for early identification and intensive care of visual disorders in KS patients. We have reported for the first time that truncating versus nontruncating mutation leads to reduced IQ in KS patients, which points to some clues regarding the molecular basis underlying intellectual disability in KS.

\section{FUNDING}

Part of this work has been supported by the French Ministry of Health (Programme Hospitalier de Recherche Clinique national AOM 07-090), Fondation Maladies Rares, and the French Kabuki Association.

\section{CONFLICT OF INTEREST}

The authors declare no conflict of interest

\section{REFERENCES}

1. Niikawa N, Kuroki Y, Kajii T, et al. Kabuki make-up (Niikawa-Kuroki) syndrome: A study of 62 patients. American Journal of Medical Genetics 1988: 31: 565-589.

2. Kuroki Y, Suzuki Y, Chyo H, Hata A, Matsui I. A new malformation syndrome of long palpebral fissures, large ears, depressed nasal tip, and skeletal anomalies associated with postnatal dwarfism and mental retardation. J Pediatr. 1981 : 99 :570-573.

3. Niikawa N, Matsuura N, Fukushima Y, Ohsawa T, Kajii T. Kabuki make-up syndrome: a syndrome of mental retardation, unusual facies, large and protruding ears, and postnatal growth deficiency. J Pediatr. 1981 : 4:565-9.

4. Ng SB, Bigham AW, Buckingham KJ, et al. Shendure J.Exome sequencing identifies MLL2 mutations as a cause of Kabuki syndrome. Nat Genet. 2010 : 42:790-3. 
5. Banka S, Veeramachaneni R, Reardon W, et al. How genetically heterogeneous is Kabuki Syndrome? MLL2 testing in 116 patients, review and analyses of mutation and phenotypic spectrum. European Journal of Human Genetics. 2012: 20: 381-8

6. Lederer D, Grisart B, Digilio MC, et al. Deletion of KDM6A, a histone demethylase interacting with MLL2, in three patients with Kabuki syndrome. Am J Hum Genet. 2012 : 90:119-24.

7. Banka S, Lederer D, Benoit V, et al. Novel KDM6A (UTX) mutations and a clinical and molecular review of the X-linked Kabuki syndrome (KS2): Novel KDM6A mutations and review of X-linked KS. Clinical Genetics 2015: 87: 252-258.

8. Bögershausen N, Wollnik B. Unmasking Kabuki syndrome: Unmasking Kabuki Syndrome. Clinical Genetics 2013: 83: 201-211.

9. Matsumoto N, Niikawa N. Kabuki make-up syndrome: a review. Am J Med Genet C Semin Med Genet 2003: 117: 57-65.

10. Vaux KK, Jones KL, Jones MC, Schelley S, Hudgins L. Developmental outcome in Kabuki syndrome. Am J Med Genet A 2005: 132: 263-264.

11. Morgan AT, Mei C, Da Costa A, et al. Speech and language in a genotyped cohort of individuals with Kabuki syndrome. American Journal of Medical Genetics Part A 2015: 167: 1483-1492.

12. Mervis CB, Becerra AM, Rowe ML, Hersh JH, Morris CA. Intellectual abilities and adaptive behavior of children and adolescents with Kabuki syndrome: a preliminary study. Am J Med Genet A 2005: 132A: 248-255.

13. Bögershausen N, Gatinois V, Riehmer V, et al. Mutation Update for Kabuki Syndrome Genes KMT2D and KDM6A and Further Delineation of X-Linked Kabuki Syndrome Subtype 2. Hum Mutat. 2016 : 37:847-64. 
14. Wechsler, D. WISC-IV technical and interpretive manual. San Antonio: Psychological Corporation, 2003.

15. Martens MA, Wilson SJ, Reutens DC. Research Review: Williams syndrome: a critical review of the cognitive, behavioral, and neuroanatomical phenotype. Journal of Child Psychology and Psychiatry 2008: 49: 576-608.

16. Antshel KM, AbdulSabur N, Roizen N, Fremont W, Kates WR. Sex Differences in Cognitive Functioning in Velocardiofacial Syndrome (VCFS). Developmental Neuropsychology 2005: 28: 849-869.

17. Tsai M-L, Hung K-L, Tsan Y-Y, William T-HT. Long-term neurocognitive outcome and auditory event-related potentials after complex febrile seizures in children. Epilepsy \& Behavior 2015: 47: 55-60.

18. Turner C, Lachlan K, Amerasinghe N, et al. Kabuki syndrome: new ocular findings but no evidence of 8p22-p23.1 duplications in a clinically defined cohort. European Journal of Human Genetics 2005: 13: 716-720

19. Wong HT, McCartney DL, Lewis JC, Sampson JR, Howe CJ, de Vries PJ. Intellectual ability in tuberous sclerosis complex correlates with predicted effects of mutations on TSC1 and TSC2 proteins. Journal of Medical Genetics 2015: 52: 815-822.

\section{TITLES AND LEGENDS TO FIGURES}

Table 1: Clinical and molecular features of Kabuki syndrome (KS) children.

Table 2: IQ scores, indexes and subtest scores obtained by the Weschler Intelligence Scale for Children, fourth edition (WISC-IV) in KS patients. 
Table 3: WISC-IV scores by clinical and molecular data for KS patients.

Table 4: Scores for subtests of the WISC-IV by clinical features and presence of heterogeneous intellectual profile (HIP; i.e., WMI>PRI) in KS patients.

Fig. 1: Close-up view on the exon 48 and schematic representation of the related protein domains.
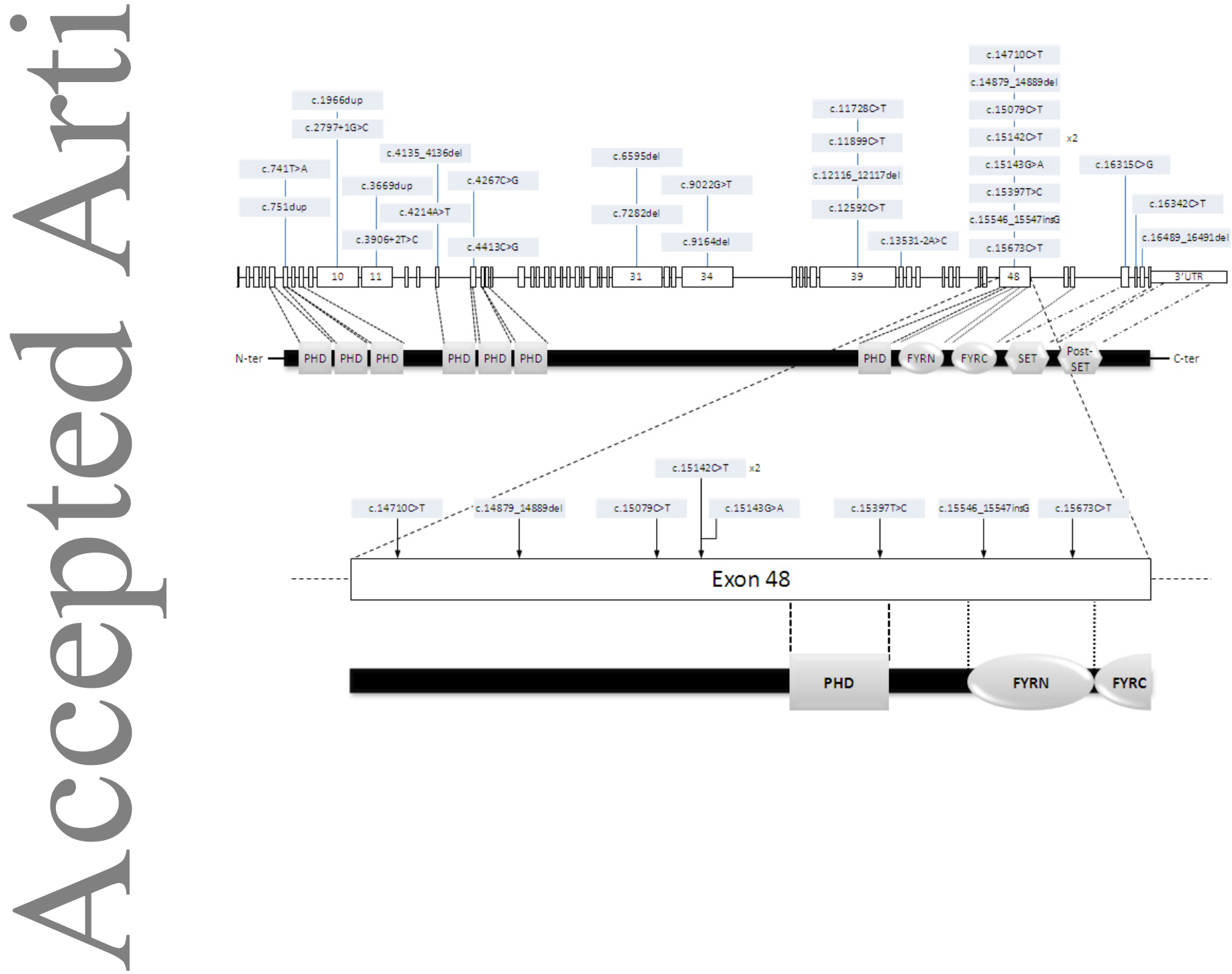
Fig. 2: Comparison of indexes of the WISC-IV.

The VCI-WMI scores were higher than PRI-PSI scores. The comparison between the WMIVCI scores and between PRI-PSI scores showed only small differences.
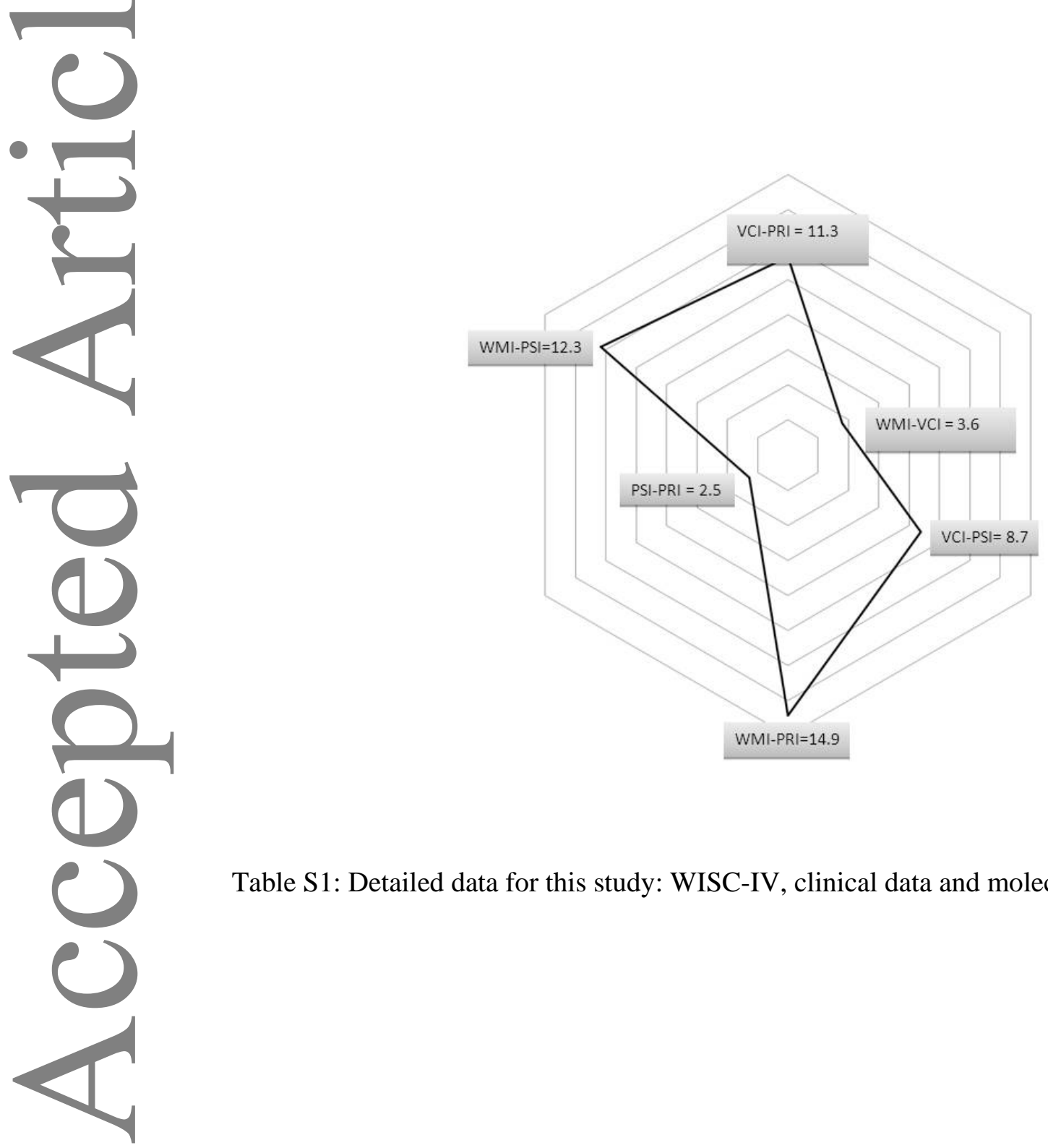

Table S1: Detailed data for this study: WISC-IV, clinical data and molecular data. 
Table 1.

Patients with KS (n=31)

\begin{tabular}{lc}
\hline Demographic data & \\
\hline Age & $11.48(2.77)$ \\
Gender (male) & $16 / 31(51,6)$ \\
\hline Clinical data & $5 / 26(19.23)$ \\
\hline Cleft Palate & $4 / 26(15.38)$ \\
Epilepsy & $7 / 26(26.92)$ \\
Microcephaly & $10 / 26(38.46)$ \\
Heart anomaly & $10 / 21(47.62)$ \\
Ophtalmic anomaly & $5 / 22(22.73)$ \\
Hearing impairment & \\
\hline Molecular data & $21 / 31(67.74)$ \\
\hline Truncating mutation & $10 / 31(32.26)$ \\
\hline Non-truncating mutation &
\end{tabular}

Values are mean (SD) or number of patients/total number of patients (\%). 
Table 2.

\begin{tabular}{llll}
\hline Index/ Subtest & n & Mean (SD) & Range \\
\hline FSIQ & 31 & $57.4(14.6)$ & $40-103$ \\
\hline Indexes & & & \\
\hline \multicolumn{1}{l}{ VCI } & 31 & $71.2(17.1)$ & $45-106$ \\
$\quad$ PRI & 31 & $59.9(13.8)$ & $45-109$ \\
WMI & 31 & $74.8(19.2)$ & $50-115$ \\
$\quad$ PSI & 31 & $62.5(10.6)$ & $50-86$ \\
\hline Subtests & & & \\
\hline Block design & 26 & $2.77(3.0)$ & $1-12$ \\
Similarities & 26 & $7.23(3.4)$ & $1-14$ \\
$\quad$ Digit span & 26 & $6.96(3.6)$ & $1-14$ \\
$\quad$ Picture concepts & 26 & $4.5(2.9)$ & $1-11$ \\
Coding & 26 & $1.85(1.5)$ & $1-7$ \\
Vocabulary & 26 & $4.77(3.2)$ & $1-11$ \\
$\quad$ Letter number & 24 & $5.17(3.1)$ & $1-11$ \\
sequencing & 26 & $3.58(2.7)$ & $1-11$ \\
$\quad$ Matrix reasoning & 26 & $4.65(2.9)$ & $1-12$ \\
Comprehension & 26 & $4.21(3.0)$ & $1-10$ \\
Symbol search & 24 & $2.2(2.7)$ & $1-7$ \\
Picture completion & 5 & $1.67(1.0)$ & $1-3$ \\
Cancellation & 6 & $2.14(1.7)$ & $1-5$ \\
Information & 7 & $3.14(2.9)$ & $1-8$ \\
Arithmetic & 7 & $8(-)$ & 8 \\
$\quad$ Word reasoning & 1 & & \\
\hline
\end{tabular}

SD: standard deviation; FSIQ:Full Scale Intellectual Quotient; VCI: verbal comprehension index; PRI: Perceptual Reasoning Index; WMI: Working Memory Index; PSI: Processing Speed Index 
Table 4.

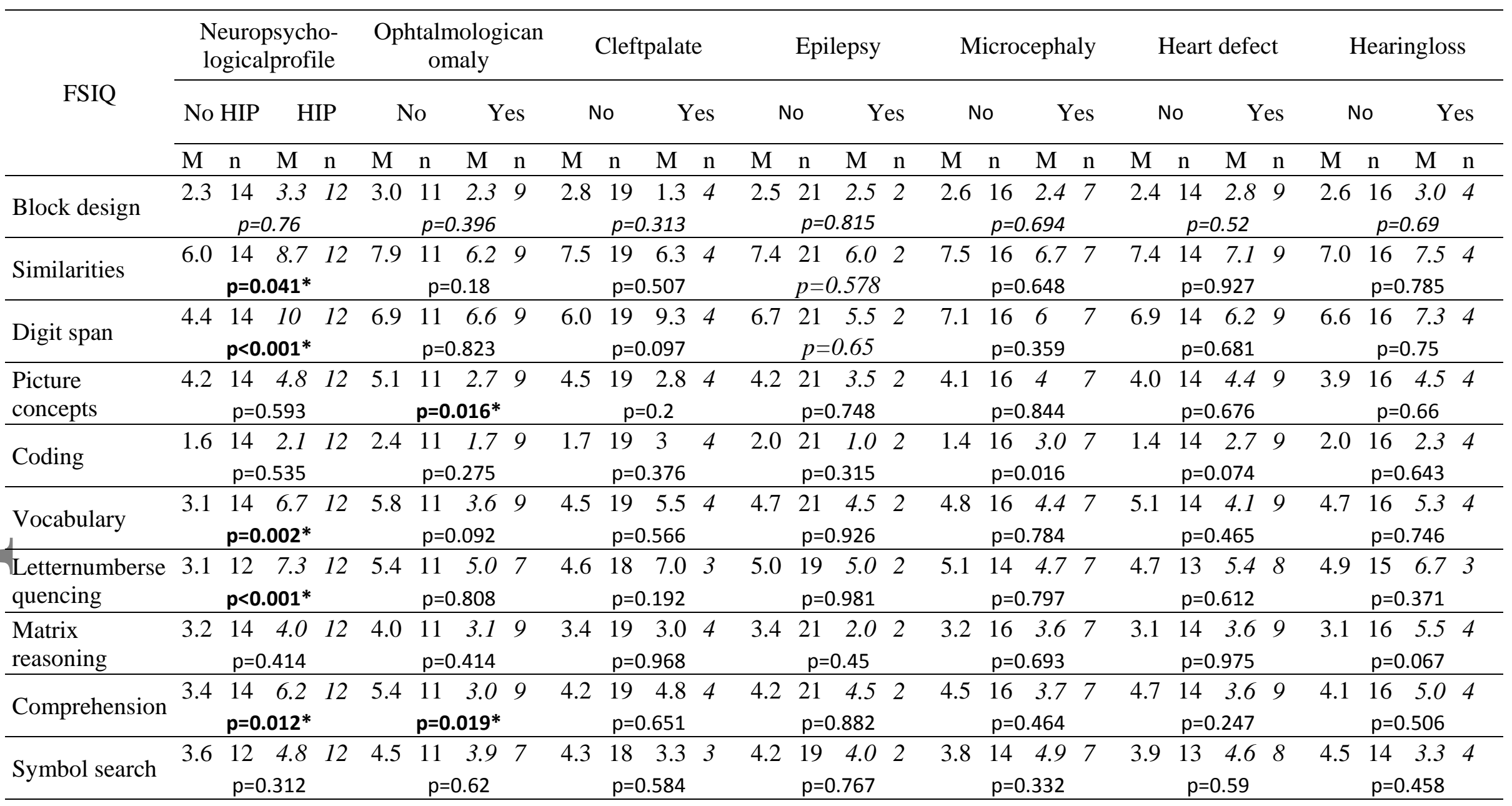




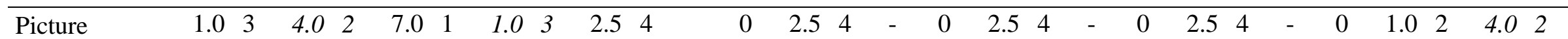
completion $\quad \mathrm{p}=0.272 \quad \mathrm{p}<0.001 * \quad \mathrm{p}=0.423$

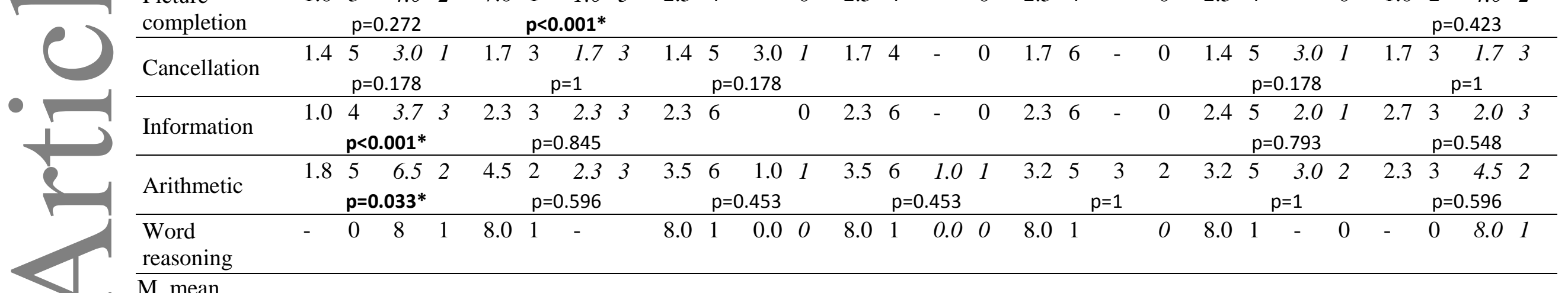
M, mean 